Nicholas Tarlov, MD

Yih Lin Nien, MD

Osama O. Zaidat, MD, MS

Thanh N. Nguyen, MD

Correspondence \& reprint requests to Dr. Nguyen: thanh.nguyen@bmc.org

\section{Periprocedural management of acute ischemic stroke intervention}

\section{ABSTRACT}

Periprocedural medical management is an important aspect in optimizing the outcome of patients who undergo endovascular treatment for acute ischemic stroke. Blood pressure, fluid hydration, and antithrombotics are some of the elements that need to be tailored carefully to the patient according to the patency of his or her cerebral vasculature, the extent of his or her infarct, and the potential for hemorrhagic transformation. This article reviews the medical care of acute stroke patients before and after endovascular therapy. Neurology ${ }^{\circledR}$ 2012;79 (Suppl 1):S182-S191

\section{GLOSSARY}

AHA = American Heart Association; ASPECTS = Alberta Stroke Program With Early CT Score; DWI = diffusion-weighted imaging; ECASS = European Cooperative Acute Stroke Study; IA = intra-arterial; $\mathbf{M C A}=$ middle cerebral artery; $\mathbf{M E R C I}=$ Mechanical Embolus Removal in Cerebral Ischemia; MR = magnetic resonance; MR RESCUE = Mechanical Retrieval and Recanalization of Stroke Clots Using Embolectomy study; NEST = Neurotherma Effectiveness and Safety Trial; NIHSS = NIH Stroke Scale; NINDS = National Institute of Neurological Disorders and Stroke; PROACT = Prolyse in Acute Cerebral Thromboembolism; tPA = tissue plasminogen activator.

Intra-arterial (IA) treatment of stroke has made important strides in advancing acute stroke therapy in the past 2 decades. The Prolyse in Acute Cerebral Thromboembolism (PROACT) II trial was the first study demonstrating benefit of IA urokinase in comparison with IV heparin in patients with middle cerebral artery (MCA) occlusion presenting within 6 hours. ${ }^{1}$ The Interventional Management of Stroke II trial showed that endovascular treatment improved outcome after acute stroke in comparison with historical controls from the National Institute of Neurological Disorders and Stroke (NINDS) trial of tissue plasminogen activator (tPA). ${ }^{2}$ Similarly, pooled data from the Mechanical Embolus Removal in Cerebral Ischemia (MERCI) and multi-MERCI registries suggested that recanalization of a large-vessel occlusion is associated with improved outcomes. ${ }^{3,4}$ The Interventional Management of Stroke III trial compared IV tPA to IV tPA bridging to endovascular therapy in patients with acute stroke presenting within 3 hours. ${ }^{5}$ Trial enrollment was held in April 2012. There were no serious safety concerns and further analysis is pending. The Mechanical Retrieval and Recanalization of Stroke Clots Using Embolectomy (MR RESCUE) trial was recently completed, comparing mechanical thrombectomy with best medical management in patients with ischemic stroke and large-vessel occlusion presenting within 8 hours of onset of symptoms. The results of these trials will be an important step forward in providing evidence that endovascular therapy improves outcomes.

Despite the plethora of past and ongoing studies on IA stroke therapy, the literature on periprocedural medical management for these patients is sparse. Attention to antithrombotic management, blood pressure control, hydration, and neuroimaging state are important details that can impact patient outcome. The purpose of this article is to provide an overview of major aspects of the management of acute ischemic stroke before, during, and after endovascular interventions. The evidence to support the periprocedural management of these cases is largely based on a case series level of data. Although several of these topics are discussed elsewhere in

From the Departments of Neurology (N.T., Y.N., T.N.N.), Neurosurgery (T.N.N.), and Radiology (N.T., T.N.N.), Boston Medical Center, Boston, MA; and Departments of Neurology, Neurosurgery, and Radiology, Medical College of Wisconsin (O.O.Z.), Milwaukee, WI.

Go to Neurology.org for full disclosures. Disclosures deemed relevant by the authors, if any, are provided at the end of this article. 
this supplement, this article highlights patient selection, neuroimaging, medical management, and novel therapies that are currently under investigation.

PREPROCEDURAL MANAGement Proper selection and rapid triage of patients with acute stroke as candidates for IA treatment is important in order to salvage as much at-risk brain as possible. Delays in patient presentation or transfer from a referring hospital may be a contributing factor to the ineligibility of stroke patients for endovascular stroke treatment. In one study, every minute of delay in patient transfer decreased the chances of being treated with IA therapy by $2.5 \% .^{6}$ In patients who are being referred to an endovascular center, pretransfer notification of the stroke, neuroradiology, and neuroangiography teams of a candidate for intervention may reduce vital minutes from door to needle and reperfusion time. A medical record number could be created ahead of time and the patient could be registered prior to arrival. Obtaining the patient's medical history, family contact, laboratory values (creatinine, coagulation parameters, complete blood cell count, EKG), and CT scan results in advance can help prepare and triage management at the recipient institution. The neurologic evaluation should begin rapidly at arrival, while the patient's airway, breathing, and circulation are being evaluated in the emergency department.

The vascular neurologist's evaluation is important for deciding whether or not to attempt endovascular therapy in a patient with an acute ischemic stroke. A brief history and physical examination establish the time of symptom onset, probable location of the ischemic insult, and severity of the stroke. An NIH Stroke Scale (NIHSS) score provides an estimate of the stroke severity and probability of large-vessel occlusion. ${ }^{7}$ A higher NIHSS score is more likely to correlate with the presence of a proximal vessel occlusion. ${ }^{7,8}$ The goal of the evaluation is to select patients with small volumes of core infarction, large areas of brain at risk for infarction, and significant clinical deficits. Efficient selection of patients for endovascular therapy may be a moving target because the neurologic examination findings may fluctuate greatly. The stroke and neuroangiography teams should be ready to adapt accordingly.

Blood pressure management. Augmentation of cerebral blood flow is important in the management of acute ischemic stroke patients to increase perfusion to the area of ischemia and to recruit collateral flow. This can be achieved by withholding antihypertensive treatment prior to the procedure, allowing the patient's blood pressure to rise spontaneously as part of the phys- iologic response to stroke. If a patient is a candidate for IV tPA, the goal is also for permissive hypertension, but not to exceed $185 / 105 \mathrm{~mm} \mathrm{Hg}$, because this increases the risk of hemorrhagic conversion after $\mathrm{PAA}$ and excludes the patient's candidacy for IV thrombolysis. ${ }^{9}$ In patients who are not eligible for IV or IA tPA, the 2007 acute ischemic stroke American Heart Association (AHA) guidelines recommend blood pressure should not be lowered unless it is greater than 220/120. ${ }^{10}$

Lowering the head of the bed is another strategy that may improve cerebral perfusion. ${ }^{11,12}$ In cases where a patient's clinical deficit worsens at a lower blood pressure, administration of a vasopressor may be considered, but it is not recommended for most stroke patients outside of the setting of clinical trials. ${ }^{10} \mathrm{~A}$ retrospective review of patients who were given vasopressors for acute stroke demonstrated that this practice may be safe and was not associated with hemorrhagic conversion of the infarct. ${ }^{13,14}$ However, after the first 24 to 48 hours from onset of symptoms, the likelihood of benefit from induced hypertension waned as the territory of the ischemic penumbra evolved into infarct. Gradual lowering of blood pressure should be undertaken. If a vasopressor agent is used in management of ischemic stroke, careful monitoring is essential, as induced hypertension may cause myocardial ischemia due to increased afterload and myocardial demand, leading to myocardial infarction. Continuous telemetry, serial cardiac enzyme, and EKG monitoring should be performed for the rare cases in which it is necessary to induce hypertension.

Hydration. Proper hydration is important in acute stroke patients, particularly those who are being considered for contrast studies such as CT angiography or catheter angiography. If the patient's blood pressure is low or the patient appears hypovolemic, the blood pressure can be increased with IV fluids. Hypotonic fluids should not be given as these may aggravate cerebral edema. Fluids containing dextrose should also be avoided unless the patient is hypoglycemic. ${ }^{10}$ Because catheters used during the procedure are continuously flushed, the patient may receive a higher amount of fluid during the embolectomy, depending on the length of the procedure. The patient's total fluids in and urine output should be monitored during the procedure so that the net fluid balance can be calculated. In patients with congestive heart failure or a low ejection fraction, it may be helpful to have a member of the anesthesia or neurocritical care team present to monitor the patient to prevent pulmonary edema.

Antithrombotic therapy. Proper administration and timing of antithrombotic agents also plays an important role in the management of the patient with acute 
ischemic stroke. In a study of IV thrombolysis, the combination of aspirin and clopidogrel at baseline was associated with a significantly higher rate of symptomatic intracranial hemorrhage. ${ }^{15}$ In patients who have received tPA, antithrombotic and antiplatelet agents should not be given for 24 hours afterward, by the NINDS study protocol, to mitigate the risk of bleeding. ${ }^{9}$ After administration of IV or IA tPA, antiplatelet therapy with aspirin (or aspirin/ dipyridamole, clopidogrel) can be given after a 24hour interval has elapsed. If the patient did not receive any tPA but only underwent mechanical thrombectomy, then an antiplatelet agent can be administered earlier after CT or MRI confirms absence of intracranial hemorrhage post-intervention.

Neuroimaging. Neuroimaging, including CT and MRI with or without perfusion, is critical in providing information to optimize medical management for the patient and to select patients for endovascular therapy. Information such as an estimate of the volume of infarct, the location of an occlusion, and the volume of viable brain at risk for infarction due to decreased blood flow is critical in orienting medical and endovascular management. A limitation of using the advanced neuroimaging techniques is the time required to perform the studies. ${ }^{16}$ It has been estimated that in a typical large-vessel supratentorial infarct, 1.9 million neurons and 12 kilometers of myelinated fibers are lost each minute. ${ }^{17}$ Information about the ischemic penumbra and volume of infarct should be determined as quickly and efficiently as possible.

CT. A noncontrast head CT is invaluable in the triage of patients to endovascular intervention. The study is widely available, offers useful information quickly, and rapidly excludes patients with intracranial hemorrhage. Loss of gray-white differentiation, obscuration of the lentiform nucleus, ${ }^{18}$ and loss of the insular ribbon ${ }^{19}$ are early signs of ischemia on CT that may be used to estimate the extent of infarction. A hyperdense MCA or basilar artery on CT can be indicative of the presence of thrombus in the appropriate clinical setting. ${ }^{20}$

An infarct greater than one-third of the MCA territory on noncontrast head CT has been proposed as a contraindication for endovascular intervention, which was based on the risk of hemorrhagic transformation after IV tPA that was seen in patients with large infarcts in the European Cooperative Acute Stroke Study (ECASS) trial. ${ }^{21}$ The ECASS trial randomized patients to $1.1 \mathrm{mg} / \mathrm{kg}$ of IV tPA or placebo. Von Kummer et al. ${ }^{22}$ prospectively evaluated the pretreatment noncontrast head CTs of patients in both arms of the trial and categorized them as having no hypoattenuation, hypoattenuation in less than $1 / 3$ of the MCA territory, or hypoattenuation in greater than $1 / 3$ of the MCA territory. Patients with hypodensity in greater than $1 / 3$ of the MCA territory experienced no beneficial effect of IV tPA and had an increased risk of fatal brain hemorrhage. Patients with infarct in less than $1 / 3$ of the MCA territory experienced benefit from IV tPA and had a decreased risk of hemorrhagic conversion of the infarct. Ideally, CT studies should take place within 1 hour of the endovascular intervention to maximize the chances of detecting an area of infarction and the presence of hemorrhagic transformation prior to endovascular intervention. ${ }^{16}$

Use of ASPECTS. Visual estimation of the size of the infarct can be difficult to ascertain and reproduce consistently. A more reliable method of identifying the extent of an infarction is the use of the Alberta Stroke Program With Early CT Score (ASPECTS). ${ }^{23}$ An ASPECTS is calculated by counting 10 standardized areas on a noncontrast head CT and deducting a point for each area with hypoattenuation. A higher ASPECTS correlates with a low area of infarction and vice versa. ASPECTS were shown to provide greater interrater reliability in quantifying the extent of early ischemic changes, compared with visual estimation of identifying an infarct greater than $1 / 3$ of the MCA territory. ${ }^{23}$ A low ASPECTS (less than 7) has been validated in a retrospective study ${ }^{24}$ as a useful tool for predicting which patients are less likely to have a good outcome after mechanical thrombectomy and which patients are more likely to have hemorrhagic conversion of infarct after IV thrombolytics. ${ }^{25}$ In a retrospective study of data from the PROACT II trial, an ASPECTS greater than 7 correlated with improved functional outcome in patients with MCA occlusion undergoing thrombolysis with IA prourokinase. ${ }^{26}$

CT perfusion. The goal of CT perfusion is to differentiate between brain tissue with cerebral blood flow so low that it is likely to be already infarcted (the "infarct core") and surrounding areas of brain still viable but likely to infarct if sufficient blood flow is not restored (the "ischemic penumbra"). Thresholds for blood flow have been used to estimate volume of the infarct core and ischemic penumbra, but these have not been validated in prospective trials, as many of these parameters may differ between patients. Also, definitions of the ischemic penumbra and infarct core differ between studies of CT perfusion, and protocols for these studies are not standardized across institutions. ${ }^{27,28}$ The reliable use of perfusion studies is also made difficult in patients who have contralateral stenosis or occlusion, patients with cardiac failure, and patients who are agitated or moving during imaging acquisition. 
MRI. Emergent MRI can rapidly detect the core of the infarct with a diffusion-weighted imaging (DWI) sequence or location of an occlusion of proximal intracranial arteries by magnetic resonance angiography. The presence of hemorrhage, either acute or occult, can be detected with gradient echo with high sensitivity compared with CT. ${ }^{29}$ The application of ASPECTS to DWI may also predict hemorrhagic risk after IA thrombolysis, as shown in one retrospective study. ${ }^{30}$ DWI lesion volume can also be used to decide whether to administer endovascular therapy; a DWI lesion $\leq 70 \mathrm{~mL}$ correlated with better outcome in patients who received IA thrombolysis in one study. ${ }^{31}$

Magnetic resonance (MR) perfusion can provide information similar to that obtained with CT perfusion, but with the added benefit of defining the infarct core with greater reliability, merely by DWI sequence. A disadvantage of MR perfusion, and MRI in general, is the increased time required to perform the study. ${ }^{16}$ Although many neuroradiologists claim that MRIs can be obtained in fifteen minutes, in our experience it usually takes about 30 minutes if you include the time required to transport the patient to MRI and move them onto and off of the MRI table.

One of the most efficient methods of selecting patients is to use the concept of clinical diffusion mismatch. ${ }^{32}$ A patient presenting with a high NIHSS score is expected to have a large territory of infarction or ischemic penumbra. Diffusion-weighted MRI, if it can be performed within 15 to 30 minutes, can define the core infarct rapidly. A high NIHSS score (greater than 8 ) with a relatively small area of DWI abnormality $(\leq 25 \mathrm{~mL}$ ) may indicate a clinical diffusion mismatch, which has high specificity in predicting infarct growth and the presence of an ischemic penumbra. ${ }^{32,33}$

Using MRI and CT to select patients for embolectomy when the time of onset is unknown. It is possible that a subset of stroke patients who present greater than 8 hours after the onset of symptoms with an imagingdefined ischemic penumbra may benefit from mechanical clot retrieval. ${ }^{34}$ Mechanical thrombectomy may be safe in these patients who are selected with MRI or CT perfusion. ${ }^{35}$ The MR RESCUE study is currently identifying whether presence of a CT- or MRI-defined penumbra can identify patients who benefit from mechanical embolectomy, regardless of the size of the infarct.

Collateral blood flow. The presence of collateral blood flow prior to endovascular treatment is not typically used to guide endovascular therapy selection. However, its absence may indicate an area of brain destined to infarct. ${ }^{36}$ The presence of collaterals may prevent further infarction until recanalization occurs and is associated with improved outcome after IV and IA thrombolysis. ${ }^{37}$ The presence of a greater degree of collateral blood flow to an area of ischemia has also been shown to be associated with a higher rate of recanalization in a retrospective study of 222 patients. $^{38}$

INTRAPROCEDURE MANAGEMENT Every effort should be made to reduce the time for pertinent laboratory study results to become available, the time to IV tPA administration, and the time for preparation of the angiography suite. If the patient is a candidate for intervention, he or she should be directly transferred from the emergency department to CT or MRI and then to the angiography suite. Goal time from door to IV tPA has been recommended to be less than 60 minutes for eligible candidates. In endovascular therapy, similar benchmarks of time have not been established.

General anesthesia is often considered but may cause hypotension during induction, furthering cerebral ischemia. Waiting for the patient to be intubated may also prolong the time to recanalization. If conscious sedation is used instead of general anesthesia, there is concern among some interventionalists that patients may be more likely to move during the procedure, which could increase the risk of microcatheter or microwire perforation of an intracerebral blood vessel. However, in a retrospective multicenter study, patients who were given conscious sedation for acute ischemic stroke intervention were not at a higher risk for intracranial hemorrhage. There was also lower mortality and better clinical outcomes in nonintubated patients vs patients treated with general anesthesia. ${ }^{39}$ Similarly, another retrospective study found that performance of the procedure in the nonintubated state was associated with a smaller final infarct volume, lower mortality, and a better chance of a good outcome. ${ }^{40}$ Further prospective randomized studies are needed to confirm that better outcomes are a result of not intubating the patient rather than inherent imbalances in the comparison of patients who are intubated or not.

Antiplatelet therapy. Intracranial stenting for acute ischemic stroke has been offered at some centers and may be associated with a high rate of recanalization, ${ }^{41,42}$ but long-term follow-up of the mortality and outcomes is needed to evaluate for restenosis and recurrent ischemic symptoms. If placement of an intracranial stent is anticipated, adjuvant clopidogrel and aspirin is often given to prevent stent thrombosis and is continued for 3 months after placement, followed by aspirin indefinitely. In the acute phase, the timing and dosage of the medications vary among 
neurointerventionalists. Glycoprotein IIb/IIIa inhibitors are favored by some neurointerventionalists prior to or during intracranial stent placement but carry a risk of hemorrhagic transformation. ${ }^{43}$ With the high recanalization rates associated with the use of removable embolectomy and stentriever devices, intracranial stenting for acute ischemic stroke should be considered as a measure of last resort so as not to leave the patient with a foreign body and expose the patient to the risks of dual antiplatelet therapy.

Blood pressure goal. During the procedure, target blood pressure within $10 \%$ to $20 \%$ of the blood pressure on admission to the emergency department is a reasonable goal prior to recanalization. As previously mentioned, if a patient received IV tPA prior to intervention, then blood pressure maintenance should follow NINDS guidelines of less than 185/105 mm Hg. ${ }^{9}$ A systolic blood pressure consistently greater than $180 \mathrm{~mm} \mathrm{Hg}$ or diastolic pressure greater than $100 \mathrm{~mm} \mathrm{Hg}$ was an exclusion criterion for IA prourokinase in the PROACT II study. ${ }^{1}$ If IA lysis is planned, then blood pressure should be maintained below these goals to prevent reperfusion hemorrhage or hemorrhagic transformation of infarct.

POSTPROCEDURE MANAGEMENT Blood pressure. Patients should be monitored in a neurologic intensive care unit, where blood pressure should be recorded at frequent intervals. The optimum blood pressure after the procedure has not been formally studied, but it should be titrated according to the degree of arterial recanalization and the patient's neurologic examination. If full recanalization and good neurologic recovery are achieved, then goal blood pressure may be lowered to a systolic 120 to $140 \mathrm{~mm} \mathrm{Hg}$ to prevent reperfusion hemorrhage. However, opinions vary among interventionalists regarding the target blood pressure after recanalization; blood pressures of up to $180 / 105 \mathrm{~mm} \mathrm{Hg}$ in patients who have been recanalized but have not received IV tPA are recommended by some. ${ }^{44}$ If there is partial recanalization, then blood pressure could be permitted up to $185 \mathrm{~mm} \mathrm{Hg}$ systolic for 24 to 48 hours. Allowing the blood pressure to spontaneously rise to optimize collateral blood flow may also help to clear emboli from distal vessels. ${ }^{45}$ In most patients, blood pressure spontaneously decreases within days after admission without any medical treatment. ${ }^{46}$ With time, a benefit of secondary stroke prevention has been seen in stroke patients who have their blood pressure lowered over days or weeks after stroke to a normal range, defined as less than $120 / 80 \mathrm{~mm} \mathrm{Hg} .{ }^{47}$ Thus, in many cases, a normal blood pressure will be the goal after several days or weeks.
Antithrombotics. Whenever possible after the procedure, the groin sheath should be promptly removed to avoid thrombotic complications such as lowerlimb ischemia. If heparin is used during the procedure, most interventionalists would not reverse with protamine unless bleeding occurred, because reversal of heparin may be associated with stroke. ${ }^{48}$ Unfractionated heparin is administered after intervention in some centers, depending on the degree of residual clot burden, and this practice was allowed after embolectomy in the MERCI trial at the discretion of the treating physician. ${ }^{4}$ Data to support this practice are inadequate, and the AHA clinical guidelines do not discuss the administration of heparin after embolectomy. ${ }^{10}$ For large strokes, the risk of hemorrhagic conversion while on heparin is greater, and heparin is therefore contraindicated. ${ }^{10}$ Administration of deep venous thrombosis prophylaxis is independently associated with an improved outcome after acute stroke. ${ }^{49}$ Prophylactic subcutaneous heparin should be given to this high-risk population to prevent deep venous thrombosis once a neuroimaging study confirms absence of hemorrhage or after 24 hours has elapsed from tPA administration. Pneumatic compression devices can be used on the legs immediately after the patient leaves the interventional suite.

Dyslipidemia. The effect of HMG Co-A reductase inhibitor (statin) administration in acute stroke patients undergoing endovascular intervention has not been adequately studied. One study of a prospectively maintained database of patients undergoing IA fibrinolysis and mechanical thrombectomy did not find an association of statin use with increased frequency of recanalization..$^{50}$ In the same study, statin use at the time of mechanical thrombectomy was associated with a 6.5-point decrease in the NIHSS score at the time of discharge. In patients who were already on statins at the time of their large-vessel ischemic stroke, greater collateralization of blood vessels was seen prior to endovascular intervention in a retrospective study. ${ }^{51}$

Statins are not known to have an immediate effect on hemodynamics in acute ischemic stroke patients, but it is reasonable to start them during admission because of their beneficial effects on lowering the incidence of future cardiovascular events. The Stroke Prevention by Aggressive Reduction of Cholesterol (SPARCL) study showed that atorvastatin, $80 \mathrm{mg}$ daily, started within 1 to 6 months of an acute stroke, reduced the incidence of future stroke and cardiovascular events and improved outcomes with recurrent ischemic stroke. ${ }^{52,53}$ Therefore, a statin is recommended for stroke patients with a low-density lipoprotein level $>100 \mathrm{mg} / \mathrm{dL}$. In June 2011, the US Food and Drug Administration issued a warning that 
the $80-\mathrm{mg}$ dose of simvastatin should not be prescribed to patients who are not already taking this dose, because it is associated with a high risk of myopathy, especially within the first 12 months of use. ${ }^{54}$ This recommendation was derived from a comprehensive review of clinical trial data from the agency's Adverse Event Reporting System. Other HMG Co-A reductase inhibitors may be used as substitutes for the high dose of simvastatin if the lower doses are not effective.

Fever. Hyperthermia should be avoided in the acute phase because it potentiates injury to the ischemic brain and is associated with worse clinical outcomes after stroke. ${ }^{55}$ The mechanism by which fever causes worsened outcomes may be related to increased levels of GABA and glycine in febrile patients ${ }^{56}$ or to increased free radical production in febrile patients. ${ }^{56,57}$ The 2007 AHA guidelines suggest that cooling stroke patients with fever might be beneficial. ${ }^{10}$

Hyperglycemia. The presence of hyperglycemia in acute ischemic stroke patients at the time of admission is associated with worse clinical outcome and higher risk for symptomatic intracranial hemorrhage, regardless of whether or not IV thrombolysis was performed. ${ }^{58}$ Similarly, Kase et al. ${ }^{59}$ found that hyperglycemia at the time of stroke onset was associated with a higher risk of intracranial hemorrhage after IA thrombolysis. The reason for the association of hyperglycemia with a worse outcome after stroke is unclear, but tissue acidosis, lactic acidosis, free radical production, altered blood-brain barrier permeability, and development of brain edema are possible explanations. ${ }^{10}$ In patients with acute ischemic stroke undergoing endovascular intervention, higher blood glucose on admission and failure of glucose levels to decrease in the first 48 hours were associated with higher mortality and worse outcome at 90 days. ${ }^{60}$ Hallevi et al. ${ }^{61}$ showed that hyperglycemia in stroke patients undergoing endovascular intervention at the time of admission is associated with a worse outcome at the time of discharge. The cause of this relationship is unclear. It is unknown whether administration of insulin with tight glycemic control can improve outcomes, but in one pilot randomized study of acute stroke patients, this was found to be feasible and safe, although the group with the tightest glycemic control $(70-110 \mathrm{mg} / \mathrm{dL})$ had a $30 \%$ rate of hypoglycemic episodes. ${ }^{62}$ Staszewski et al. ${ }^{63}$ demonstrated that a 24-hour continuous insulin infusion to keep blood glucose at $81-126 \mathrm{mg} / \mathrm{dL}$ resulted in a 3 -point decrease in the NIHSS score at 30 days.

Other. Administration of a swallow evaluation is independently associated with an improved outcome after acute stroke. ${ }^{49} \mathrm{~A}$ bedside swallow examination should be done on admission and may need to be repeated after sedation from the procedure wears off. If the patient cannot safely swallow, a nasogastric tube may be inserted (but delayed for 24 hours if the patient received tPA). If needed, tube feeds should be started early because protein energy malnutrition after stroke is associated with an increased rate of bedsores and infections and a worse outcome. ${ }^{64,65}$ Aspiration precautions should be undertaken in all patients.

Communication. An important and sometimes overlooked aspect of periprocedural care for stroke patients is the need to communicate with the patient's family on the gravity of the stroke, expected natural history, and procedural risks. Following the procedure, the family should be updated about the results of the procedure and any changes in the patient's neurologic status. One of the most common reasons that physicians are sued is that families feel that the physician did not talk openly, did not warn them about long-term problems, or that information was communicated poorly. ${ }^{66}$

\section{FUTURE DIRECTIONS IN STROKE CARE Sev-} eral emerging technologies such as ultrasound, infrared laser, the Neuroflo catheter, ${ }^{67}$ and medications are being studied in acute stroke patients. While they may not replace standard mechanical thrombectomy and IA tPA as a means of revascularization, it is possible that in the future they may improve outcomes when used as an adjunct to standard endovascular procedures.

Ultrasound. Multiple randomized and nonrandomized studies have shown that ultrasound facilitates the lysis of blood clots in patients who are administered tPA. ${ }^{68,69}$ Ultrasound has been shown in vitro to increase the penetration of tPA into blood clot. ${ }^{70}$ Catheter-based ultrasound delivery has undergone testing in a pilot study with the EKOS catheter and is being tested in human trials. ${ }^{2}$ Microbubbles are small lipid particles that can be used in conjunction with ultrasound to enhance lysis of IA thrombus. ${ }^{71}$ Microbubbles increase the turbulence created by ultrasound around the clot. The bubbles combined with ultrasound are thought to increase the microfragmentation of the clot and increase the surface area of clot for tPA to act upon. ${ }^{72}$

Infrared laser. Infrared laser may stimulate adenosine triphosphate formation in mitochondria and prevent cell death in the ischemic penumbra. ${ }^{73,74}$ The Neurotherma Effectiveness and Safety Trial (NEST-1) was a randomized controlled study of infrared laser applied, on average, 16 hours after onset of stroke. The trial revealed that use of the laser was associated with 
reduced disability at 90 days. $^{73}$ In addition, a recent meta-analysis of data from NEST-1 and NEST-2 showed a significantly improved outcome at 90 days for patients treated with low-energy transcranial laser within 24 hours of an ischemic stroke. ${ }^{74}$ Another randomized controlled trial showed a non-statistically significant improvement in outcome with laser therapy started within 24 hours of ischemic stroke. ${ }^{75}$ If larger trials show a benefit, then this technology has the potential to treat many patients with stroke who are beyond the 6-hour time window at the time of their arrival in the emergency department.

Neuroprotection. Theoretically, it may be possible to block many of the enzymatic steps in the biochemical cascade that leads to neuronal cell death. However, despite the growing understanding of the biochemical mechanisms of free radical generation and the influx of calcium into cells, clinical trials of "neuroprotective agents" have not demonstrated efficacy in stroke patients. As one author has pointed out, many clinical trials have tested therapies greater than 6 hours after the onset of stroke. This may be beyond the window during which many therapies may be effective. ${ }^{76}$ Several neuroprotective agents have been proposed, including oxygen and albumin.

Although hyperbaric oxygen was not shown to be useful, high-flow oxygen at normal atmospheric pressure has been considered as a method to slow cell death after ischemic stroke. ${ }^{77-79}$ However, the routine use of oxygen is not currently recommended by the AHA for acute stroke patients. ${ }^{10}$

A phase I pilot trial in human ischemic stroke patients showed that albumin could be tolerated, with the only adverse event being pulmonary edema in a minority of subjects. ${ }^{80}$ However, final results of part 1 of the trial revealed that the 90-day death rate in patients treated with albumin within 5 hours of the onset of symptoms was higher than in patients treated with saline. ${ }^{81}$ The excess deaths were mostly in older patients with large strokes who had received large volumes of IV fluids. Increased rates of pulmonary edema and myocardial infarction also occurred in the albumin group. Changes were made in the study protocol, including imposing an upper age limit for study subjects, restricting the rate of IV fluid administration, and excluding patients with elevated troponins. The study has continued as a separate study called the ALIAS Part 2 Trial. ${ }^{81}$

Neuroflo dual aortic balloon catheter. The Neuroflo device (CoAxia, Maple Grove, MN) is an intermittently inflating balloon catheter placed in the descending aorta that may improve cerebral perfusion by augmenting flow toward the cranium rather than the splanchnic bed. The increase in cerebral perfusion pressure may enhance recruitment of collaterals, prevent arteriolar collapse, and result in salvage of penumbral tissue. ${ }^{82}$ Interestingly, in a nonstroke porcine model, the increase in cerebral blood flow persisted for 90 minutes after the aortic balloon occlusion was removed. ${ }^{83}$

The SENTIS trial of the Neuroflo device included patients presenting with strokes up to 14 hours after symptom onset, with baseline NIHSS scores between 5 and $18 .{ }^{84}$ Patients were randomized 1:1 to either Neuroflo treatment or standard medical care. The primary efficacy endpoint was a return to normal neurologic status, based on the NINDS Global Score, and the secondary efficacy measure of a modified Rankin Scale score of $0-2$ and Rankin shift. The trial did not reach statistical significance in its primary efficacy measure. However, it did achieve the trial's primary safety endpoint. A post hoc analysis showed a non-statistically significant trend toward improved neurologic outcomes, as measured by 90-day modified Rankin Scale scores of 0-2 in earlypresenting patients, patients with moderate strokes, and patients over 70 years of age. There was also a trend toward decreased mortality from all causes in patients treated with the device. Anticipated thrombolysis or thrombectomy was an exclusion criterion for the SENTIS trial.

The Neuroflo device has also been tested in patients after IV tPA. ${ }^{85}$ In a rodent stroke model, the combination of tPA and $70 \%$ occlusion of the abdominal aorta for 1 hour reduced infarct volume greater than tPA alone or aortic occlusion alone. ${ }^{82}$ There was no increase in the risk of hemorrhagic conversion of infarct with the combined use of tPA and aortic occlusion, suggesting the possibility that the Neuroflo device might be used in patients who receive $\mathrm{tPA}$ in the future.

DISCUSSION Patient selection with the aid of neuroimaging, timely endovascular intervention, and proper medical therapy are important components in the management of patients undergoing endovascular therapy for acute ischemic stroke. Future technologic developments such as ultrasound, infrared laser therapy, and partial aortic occlusion may add to our therapeutic arsenal.

\section{AUTHOR CONTRIBUTIONS}

Dr. Tarlov: drafting/revising the manuscript. Dr. Nien: drafting/revising the manuscript, acquisition of data. Dr. Zaidat and Dr. Nguyen: drafting/ revising the manuscript, study concept or design, study supervision.

\section{DISCLOSURE}

Dr. Tarlov and Dr. Nien report no disclosures. Dr. Zaidat serves on the scientific advisory board for Talecris; served on the adjudication committee for Stryker; received speaker honoraria from Stryker; served on the editorial board of Frontiers in Neurology (Endovascular \& Interventional 
Neurology Section); serves as Editor of The Journal of Neurointerventional Surgery, and serves as Associate Editor and is a member of the Editorial Board of Journal of Stroke \& Cerebrovascular Diseases; served as a consultant for Stryker Neurovascular-Commercial, Codman NeurovascularCommercial, and Microvention Inc.-Commercial; and has received research support from a Society of Vascular \& Interventional Neurology (SVIN) grant for this educational activity. Dr. Nguyen serves as Associate Editor of Frontiers of Vascular and Interventional Neurology and Editor of SVIN Newsletter The Core; and she performs intra-arterial stroke procedures. Go to Neurology.org for full disclosures.

Received July 9, 2011. Accepted in final form October 7, 2011.

\section{REFERENCES}

1. Furlan AJ, Higashida R, Wechsler L, et al. Intra-arterial prourokinase for acute ischemic stroke: the PROACT II study: a randomized controlled trial: Prolyse in Acute Cerebral Thromboembolism. JAMA 1999;282:2003-2011.

2. The IMS II Trial Investigators. The Interventional Management of Stroke (IMS) II Study. Stroke 2007;38:21272135.

3. Nogueira RG, Liebeskind DS, Sung G, et al. Predictors of good clinical outcomes, mortality, and successful revascularization in patients with acute ischemic stroke undergoing thrombectomy: pooled analysis of the Mechanical Embolus Removal in Cerebral Ischemia (MERCI) and Multi MERCI trials. Stroke 2009;40:3777-3783.

4. Smith WS, Sung G, Starkman S, et al. Safety and efficacy of mechanical embolectomy in acute ischemic stroke: results of the MERCI Trial. Stroke 2005;36: 1432-1438.

5. Khatri P, Hill MD, Palesch YY, et al, for the IMS III Investigators. Methodology of the Interventional Management of Stroke III trial. Int J Stroke 2008;130-137.

6. Prabhakaran S, Ward E, John S. Transfer delay is a major factor limiting the use of intra-arterial treatment of acute ischemic stroke. Stroke 2011;42:1626-1630.

7. Poisson SN, Nguyen-Huynh MN, Johnston SC, Furie KL, Lev MH, Smith WS. Intracranial large vessel occlusion as a predictor of decline in functional status after transient ischemic attack. Stroke 2011;42:44-47.

8. Olavarría VV, Delgado I, Hoppe A, et al. Validity of the NIHSS in predicting arterial occlusion in cerebral infarction is time-dependent. Neurology 2011;76:62-68.

9. The National Institute of Neurological Disorders and Stroke rt-PA Stroke Study Group. Tissue plasminogen activator for acute ischemic stroke. N Engl J Med 1995;333: 1581-1587.

10. Adams HP, del Zoppo G, Alberts MJ, et al. Guidelines for the early management of adults with ischemic stroke: a guideline from the American Heart Association/American Stroke Association Stroke Council, Clinical Cardiology Council, Cardiovascular Radiology and Intervention Council, and the Atherosclerotic Peripheral Vascular Disease and Quality of Care Outcomes in Research Interdisciplinary Working Groups. Stroke 2007;38:1655-1711.

11. Wojner AW, El-Mitalli A, Alexandrov A. Effect of head positioning on intracranial blood flow velocities in acute ischemic stroke: a pilot study. Crit Care Nurs Q 2002;24: 57-66.

12. Wojner-Alexander AW, Zsolt G, Chernyshev OY, Alexandrov AV. Heads down: flat positioning improves blood flow velocity in acute ischemic stroke. Neurology 2005;64: $1354-1357$.
13. Rordorf G, Cramer SC, Efird JT, Schwamm LH, Buonanno F, Koroshetz WJ. Pharmacological elevation of blood pressure in acute stroke: clinical effects and safety. Stroke 1997;28:2133-2138.

14. Rordorf G, Koroshetz WJ, Ezzeddine MA, Segal AZ, Buonanno FS. A pilot study of drug-induced hypertension for treatment of acute stroke. Neurology 2001;56:12101213.

15. Diedler J, Ahmed N, Sykora M, et al. Safety of intravenous thrombolysis for acute ischemic stroke in patients receiving antiplatelet therapy at stroke onset. Stroke 2010;41:288294.

16. Higashida RT, Furlan AJ. Trial design and reporting standards for intra-arterial cerebral thrombolysis for acute ischemic stroke. Stroke 2003;34:e109-e137.

17. Saver JL. Time is brain-quantified. Stroke 2006;37:263266.

18. Tomura N, Uemura K, Inugami A, Fujita H, Higano S, Shishido F. Early CT finding in cerebral infarction: obscuration of the lentiform nucleus. Radiology 1988;168:463467.

19. Truwit CL, Barkovich AJ, Gean-Marton A, Hibri N, Norman D. Loss of the insular ribbon: another early CT sign of acute middle cerebral artery infarction. Radiology 1990; 176:801-806

20. Bastianello S, Pierallini A, Colonnese C, et al. Hyperdense middle cerebral artery CT sign: comparison with angiography in the acute phase of ischemic supratentorial infarction. Neuroradiol 1991;33:207-211.

21. Hacke W, Kaste M, Fieschi C, et al. Intravenous thrombolysis with recombinant tissue plasminogen activator for acute hemispheric stroke: the European Cooperative Acute Stroke Study (ECASS). JAMA 1995;274:1017-1025.

22. Von Kummer R, Allen KL, Holle R, et al. Acute stroke: usefulness of early CT findings before thrombolytic therapy. Radiology 1997;205:327-333.

23. Pexman JH, Barber PA, Hill MD, et al. Use of the Alberta Stroke Program Early CT Score (ASPECTS) for assessing CT scans in patients with acute stroke. Am J Neuroradiol 2001;22:1534-1542.

24. Goyal M, Menon BK, Coutts SB, et al. Effect of baseline CT scan appearance and time to recanalization on clinical outcomes in endovascular thrombectomy of acute ischemic strokes. Stroke 2011;42:93-97.

25. Hirano T, Sasaki M, Tomura N, et al. Low Alberta Stroke Program Early Computer Tomography Score within 3 hours of onset predicts subsequent symptomatic intracranial hemorrhage in patients treated with $0.6 \mathrm{mg} / \mathrm{kg}$ alteplase. J Stroke Cerebrovasc Dis Epub 2011 Jul 5.

26. Hill MD, Rowley HA, Adler F, et al. Selection of acute ischemic stroke patients for intra-arterial thrombolysis with pro-urokinase by using ASPECTS. Stroke 2003;34: 1925-1931.

27. Eastwood JD, Lev MH, Azhari T, et al. CT perfusion scanning with deconvolution analysis: pilot study in patients with acute middle cerebral artery stroke. Radiology 2002;222:227-236.

28. Eastwood JD, Lev MH, Wintermark M, et al. Correlation of early dynamic CT perfusion imaging with whole-brain MR diffusion and perfusion imaging in acute hemispheric stroke. AJNR 2003;1869-1875.

29. Chalela JA, Kidwell CS, Nentwich LM, et al. Magnetic resonance imaging and computed tomography in emergency assessment of patients with suspected acute 
stroke: a prospective comparison. Lancet 2007;369: 293-298.

30. Singer OC, Kurre W, Humpich MC, et al. Risk assessment of symptomatic intracerebral hemorrhage after thrombolysis using DWI-ASPECTS. Stroke 2009; 40: 2743-2748.

31. Yoo AJ, Verduzco LA, Schaefer PW, Hirsch JA, Rabinov JD, Gonzalez RG. MRI-based selection for intra-arterial stroke therapy: value of pretreatment diffusion-weighted imaging lesion volume in selecting patients with acute stroke who will benefit from early recanalization. Stroke 2009; 40:2046-2054.

32. Davalos A, Blanco M, Pedraza S. The clinical-DWI mismatch: a new diagnostic approach to the brain tissue at risk of infarction. Neurology 2004;62:2187-2192.

33. Prosser J, Butcher K, Allport L, et al. Clinical-diffusion mismatch predicts the putative penumbra with high specificity. Stroke 2005;36:1700-1704.

34. Abou-Chebl A. Endovascular treatment of acute ischemic stroke may be safely performed with no time window limit in appropriately selected patients. Stroke 2010;41:19962000.

35. Jovin TG, Liebeskind DS, Gupta R, et al. Imaging-based endovascular therapy for acute ischemic stroke due to proximal intracranial anterior circulation occlusion treated beyond 8 hours from time last seen well: retrospective multicenter analysis of 237 consecutive patients. Stroke 2011; 42:2206-2211.

36. Bang OY, Saver JL, Buck BH, et al. Impact of collateral flow on tissue fate in acute ischemic stroke. J Neurol Neurosurg Psychiatry 2008;79:625-629.

37. Kucinski T, Koch C, Eckert B, et al. Collateral circulation is an independent radiological predictor of outcome after thrombolysis in acute ischemic stroke. Neuroradiology 2003;45:11-18.

38. Bang OY, Saver JL, Kim SJ, et al. Collateral flow predicts response to endovascular therapy for acute ischemic stroke. Stroke 2011;42:693-699.

39. Abou-Chebl A, Lin R, Hussain MS, et al. Conscious sedation versus general anesthesia during endovascular therapy for acute anterior circulation stroke: preliminary results from a retrospective multi-center study. Stroke 2010;41: $1175-1179$.

40. Jumaa MA, Zhang F, Ruiz-Ares G, et al. Comparison of safety and clinical and radiographic outcomes in endovascular acute stroke therapy for proximal middle cerebral artery occlusion with intubation and general anesthesia versus the nonintubated state. Stroke 2010;41:11801184.

41. Gupta R, Tayal A, Levy E, et al. Intra-arterial thrombolysis or stent placement during endovascular treatment for acute ischemic stroke leads to the highest recanalization rate: results of a multicenter retrospective study. Neurosurgery 2011;68:1618-1623.

42. Levy E, Rahman M, Khalessi AA, et al. Midterm clinical and angiographic follow up for the first FDA-approved prospective, single-arm trial of primary stenting for stroke: SARIS (Stent-Assisted Recanalization for acute Ischemic Stroke). Neurosurgery 2011;69:915-920.

43. Adams HP, Effron MB, Torner J, et al. Emergency administration of abciximab for treatment of patients with acute ischemic stroke: results of an international phase III trial: Abciximab in Emergency Treatment of Stroke Trial (AbESTT-II). Stroke 2008;39:87-99.
44. Leslie-Mazwi TM, Sims JR, Hirsch JA, Nogueira RG. Periprocedural blood pressure management in neurointerventional surgery. J Neurointervent Surg 2011;3: $66-73$.

45. Caplan LR, Hennerici M. Impaired clearance of emboli (washout) is an important link between hypoperfusion, embolism, and ischemic stroke. Arch Neurol 1998;55: 1475-1482.

46. Phillips SJ. Pathophysiology and management of hypertension in acute ischemic stroke. Hypertension 1994;23: 131-136.

47. Sacco RL, Adams R, Albers G, et al. Guidelines for prevention of stroke in patients with ischemic stroke or transient ischemic attack. Stroke 2006;37:577-617.

48. Mauney MC, Buchanan SA, Lawrence A, et al. Stroke rate is markedly reduced after carotid endarterectomy by avoidance of protamine. J Vasc Surg 1995;22:264-270.

49. Bravata DM, Wells CK, Lo AC, et al. Processes of care associated with acute stroke outcomes. Arch Intern Med 2010;170:804-810.

50. Restreppo L, Bang OY, Ovbiagele B, et al. Impact of hyperlipidemia and statins on ischemic stroke outcomes after intra-arterial fibrinolysis and percutaneous mechanical embolectomy. Cerebrovasc Dis 2009;28:384-390.

51. Ovbiagele B, Saver JL, Starkman S, et al. Statin enhancement of collateralization in acute stroke. Neurology 2007; 68:2129-2131.

52. The Stroke Prevention by Aggressive Reduction in Cholesterol Levels (SPARCL) Investigators. High-dose atorvastatin after stroke or transient ischemic attack. N Engl J Med 2006;355:549-559.

53. Goldstein LB, Amarenco P, Zivin J, et al. Statin treatment and stroke outcome in the Stroke Prevention by Aggressive Reduction in Cholesterol Levels (SPARCL) Trial. Stroke 2009;40:3526-3531.

54. US Food and Drug Administration. FDA News Release. Available at: www.fda.gov/NewsEvents/Newsroom/Press Announcements/ucm258338.htm. Accessed June 8, 2011.

55. Hajat C, Hajat S, Pankaj S. Effects of poststroke pyrexia on stroke outcome: a meta-analysis of studies in patients. Stroke 2000;31:410.

56. Ginsberg MD, Busto R. Combating hyperthermia in acute stroke: a significant clinical concern. Stroke 1998;29:529534.

57. Globus MY, Busto R, Lin B, et al. Detection of free radical activity during transient global ischaemia and recirculation: effects of intra-ischemic brain temperature modulation. J Neurochem 1995;65:1250-1256.

58. Bruno A, Levine SR, Frankel MR, et al.; NINDS rt-PA Stroke Study Group. Admission glucose level and clinical outcomes in the NINDS rt-PA Stroke Trial. Neurology 2002;59:669-674.

59. Kase CS, Furlan AJ, Wechsler LR, et al. Cerebral hemorrhage after intra-arterial thrombolysis for acute ischemic stroke: the PROACT II trial. Neurology 2001;57:16031610 .

60. Natarajan SK, Dandona P, Karmon Y, et al. Prediction of adverse outcomes by blood glucose level after endovascular therapy for acute ischemic stroke. J Neurosurg 2011;114: 1785-1799.

61. Hallevi H, Barreto AD, Liebeskind DS. Identifying patients at high risk for poor outcome after intra-arterial therapy for acute ischemic stroke. Stroke 2009; 40:17801785. 
62. Johnston KC, Hall CE, Kissela BM, Bleck TP, Conaway MR; for the GRASP Investigators. Glucose Regulation in Acute Stroke Patients (GRASP) trial: a randomized pilot trial. Stroke 2009;40:2123-2128.

63. Staszewski J, Brodacki B, Kotowicz J, et al. Intravenous insulin therapy in the maintenance of strict glycemic control in nondiabetic acute stroke patients with mild hyperglycemia. J Stroke Cerebrovasc Dis 2011;20:150-154

64. Dávalos A, Ricart W, Gonzalez-Huix F, et al. Effect of malnutrition after acute stroke on clinical outcome. Stroke 1996;27:1028-1032.

65. Ha L, Hauge T, Spenning AB, et al. Individual nutritional support prevents undernutrition, increases muscle strength and improves QoL among elderly at nutritional risk hospitalized for acute stroke: a randomized, controlled trial. Clin Nutr 2010;29:567-573.

66. Forster HP, Schwartz J, DeRenzo E. Reducing legal risk by practicing patient-centered medicine. Arch Intern Med 2002;162:1217-1219.

67. Uflacker R, Schönholz C, Papamitisakis N. Interim report of the SENTIS trial: cerebral perfusion augmentation via partial aortic occlusion in acute ischemic stroke. J Cardiovasc Surg 2008;49:715-721.

68. Tsivgoulis G, Eggers J, Ribo M, et al. Safety and efficacy of ultrasound-enhanced thrombolysis: a comprehensive review and meta-analysis of randomized and nonrandomized studies. Stroke 2010;41:280-287.

69. Alexandrov AV, Molina CA, Grotta JC, et al. Ultrasoundenhanced systemic thrombolysis for acute ischemic stroke. N Engl J Med 2004;18:351:2170-2178.

70. Francis CW, Blinc A, Lee S, Cox C. Ultrasound accelerates transport of recombinant tissue plasminogen activator into clots. Ultrasound Med Biol 1995;21:419-424.

71. Molina CA, Ribo M, Arenillas J, et al. Microbubble administration accelerates clot lysis during continuous 2 $\mathrm{MHz}$ ultrasound monitoring in stroke patients treated with intravenous tPA. Stroke 2006;37:425-429.

72. Dijkmans PA, Juffermans LJM, Musters RJP, et al. Microbubbles and ultrasound: from diagnosis to therapy. Eur J Echocardiography 2004;5:245-256.

73. Lampl Y, Zivin JA, Fisher M, et al. Infrared laser therapy for ischemic stroke: a new treatment strategy. Stroke 2007; 38:1843-1849.
74. Stemer AB, Huisa BN, Zivin JA. The evolution of transcranial laser therapy for acute ischemic stroke, including a pooled analysis of NEST-1 and NEST-2. Curr Cardiol Rep 2010;12:29-33.

75. Zivin JA, Albers GW, Bornstein N, et al. Effectiveness and safety of transcranial laser therapy for acute ischemic stroke. Stroke 2009;40:1359-1364.

76. Ginsberg MD. Current status of neuroprotection for cerebral ischemia: synoptic overview. Stroke 2009;40(suppl 1): S111-S114.

77. Kim HY, Singhal AB, Lo EH. Normobaric hyperoxia extends the reperfusion window in focal cerebral ischemia. Ann Neurol 2005;57:571-575.

78. Singhal AB, Dijkhuizen RM, Rosen BR, Lo EH. Normobaric hyperoxia reduces MRI diffusion abnormalities and infarct size in experimental stroke. Neurology 2002;58: 945-952.

79. Singhal AB, Benner T, Roccatagliata L, et al. A pilot study of normobaric oxygen therapy in acute ischemic stroke. Stroke 2005;36:797-802.

80. Ginsberg MD, Hill MD, Palesch YY, Rychborst KJ, Tamariz D. The ALIAS Pilot Trial: a dose-escalation and safety study of albumin therapy for acute ischemic stroke: physiological responses and safety results. Stroke 2006;37: 2100-2106.

81. Ginsberg M, Palesch YY, Martin RH, et al. The Albumin in Acute Stroke (ALIAS) Multicenter Trial: safety analysis of part 1 and design of part 2. Stroke 2011;42:119-127.

82. Noor R, Wang CX, Todd K, et al. Partial intra-aortic occlusion improves perfusion deficits and infarct size following focal cerebral ischemia. J Neuroimaging 2010;20: 272-276.

83. Hammer M, Jovin T, Wahr J, Heiss WD. Partial occlusion of the descending aorta increases cerebral blood flow in a non-stroke porcine model. Cerebrovasc Dis 2009;28: 406-410.

84. Shuaib A, Bornstein NM, Diener HC, et al. Partial aortic occlusion for cerebral perfusion augmentation: safety and efficacy of Neuroflo in acute ischemic stroke trial. Stroke 2011;42:1680-1690.

85. Emery DJ, Schellinger PD, Selchen D, et al. Safety and feasibility of collateral blood flow augmentation following intravenous thrombolysis. Stroke 2011;42:1135-1137. 


\section{Neurology}

\section{Periprocedural management of acute ischemic stroke intervention Nicholas Tarlov, Yih Lin Nien, Osama O. Zaidat, et al. Neurology 2012;79;S182-S191 \\ DOI 10.1212/WNL.0b013e31826958d3}

This information is current as of September 24, 2012

\section{Updated Information \& Services}

References

Citations

\section{Subspecialty Collections}

Permissions \& Licensing

Reprints including high resolution figures, can be found at: http://n.neurology.org/content/79/13_Supplement_1/S182.full

This article cites 81 articles, 50 of which you can access for free at: http://n.neurology.org/content/79/13_Supplement_1/S182.full\#ref-list1

This article has been cited by 1 HighWire-hosted articles: http://n.neurology.org/content/79/13_Supplement_1/S182.full\#\#otherar ticles

This article, along with others on similar topics, appears in the following collection(s):

All Cerebrovascular disease/Stroke

http://n.neurology.org/cgi/collection/all_cerebrovascular_disease_strok e

\section{Infarction}

http://n.neurology.org/cgi/collection/infarction

Other cerebrovascular disease/ Stroke

http://n.neurology.org/cgi/collection/other_cerebrovascular_disease_st roke

Stroke prevention

http://n.neurology.org/cgi/collection/stroke_prevention

Information about reproducing this article in parts (figures,tables) or in its entirety can be found online at:

http://www.neurology.org/about/about_the_journal\#permissions

Information about ordering reprints can be found online:

http://n.neurology.org/subscribers/advertise

Neurology ${ }^{\circledR}$ is the official journal of the American Academy of Neurology. Published continuously since 1951, it is now a weekly with 48 issues per year. Copyright Copyright $@ 2012$ by AAN Enterprises, Inc.. All rights reserved. Print ISSN: 0028-3878. Online ISSN: 1526-632X.

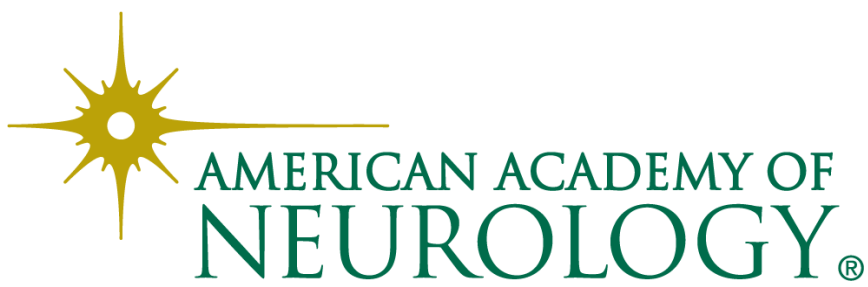

